\title{
Water Vapor Adsorption Properties of Honeycomb-Type Zeolites for Tritium Removal Systems*)
}

\author{
Masahiro TANAKA, Tatsuhiko UDA and Kenzo MUNAKATA ${ }^{1)}$ \\ National Institute for Fusion Science, 322-6 Oroshi-cho, Toki 509-5292, Japan \\ ${ }^{1)}$ Akita University, 1-1, Gakuen-machi, Tegata, Akita 010-5802, Japan
}

(Received 7 December 2010 / Accepted 17 March 2011)

\begin{abstract}
We have proposed the application of a honeycomb-type adsorbent and catalyst for an advanced tritium removal system. Honeycomb-type materials exhibit a much lower pressure drop than pellet-type materials. In this study, the water vapor adsorption properties of various types of honeycomb adsorbents were evaluated using the breakthrough method at a constant flow rate of $307 \mathrm{~cm}^{3} / \mathrm{min}$ under various temperature and water vapor partial pressure conditions. The results revealed that the adsorption capacity of water vapor on the honeycomb-type zeolite increased with the water vapor partial pressure and the zeolite content of the honeycomb adsorbents. Furthermore, the honeycomb-type zeolite was found to have a higher adsorption rate than the pellet-type zeolite, and the temperature required for regeneration of the honeycomb-type zeolite was at least $450 \mathrm{~K}$. From the viewpoint of practical use, the honeycomb-type adsorbent that contained $50 \%$ zeolite with 200 cells per square inch was considered to have superior adsorption properties and a lower pressure drop among a series of honeycomb-type adsorbents.
\end{abstract}

(c) 2011 The Japan Society of Plasma Science and Nuclear Fusion Research

Keywords: tritium removal system, high throughput, low pressure drop, honeycomb, cell density, zeolite content, water adsorption, molecular sieves, breakthrough curve

DOI: $10.1585 /$ pfr.6.2405038

\section{Introduction}

Tritium is a radioactive hydrogen isotope that decays to ${ }^{3} \mathrm{He}$ by emitting a beta particle with a half-life of 12.33 years. The maximum and average energies of the beta particle emitted from tritium are $18.6 \mathrm{keV}$ and $5.69 \mathrm{keV}$, respectively. Because it produces low-energy beta particles, tritium is usually not considered an external radiation exposure hazard. However, inhalation, ingestion, or skin absorption of tritium can result in internal radiation damage. This is because tritium oxide has a chemical behavior identical to that of water, and hence, allowing rapid uptake by the human body [1].

Moreover, tritium has a high coefficient of diffusion, allowing it to diffuse through metals, and thus, escape containment. Tritium that leaks out should be rapidly removed from the atmosphere. The most widely used atmospheric tritium removal technique is oxidation to water by catalytic oxidation reactors, followed by adsorption [2]. In this procedure, the adsorbent must be periodically regenerated to remove the water so that it can be reused; tritium (HTO) is removed as a condensate during this regeneration. Various types of adsorbents have been utilized in tritium removal systems. Among these, pellet-type molecular sieves are most common. The adsorption characteristics of water vapor and tritiated water vapor on a pellet-type molecu-

author's e-mail: tanaka.masahiro@nifs.ac.jp

*) This article is based on the presentation at the 20th International Toki Conference (ITC20). lar sieve 5A have been reported [3,4]. This conventional tritium removal system has been used in tritium handling facilities worldwide and found to have adequate performance [5-8].

However, tritium removal systems used for safety management in fusion power plants must achieve large processing volumes and throughputs and have large pressure drops. The pressure drop in the system determines the load on the pumping system. To reduce the pressure drop, a tritium removal system using a honeycombtype catalyst has been proposed. Advantages of the use of honeycomb-type materials compared with pellet-type materials include a lower pressure drop, easier maintenance, and a more uniform flow. In previous studies, we investigated the oxidation performance of a honeycomb-type catalyst for $\mathrm{H}_{2}$ and $\mathrm{CH}_{4}$ in air [9-11]. The results revealed that the honeycomb-type material produced a lower pressure drop than a pellet-type material and had nearly the same oxidation performance as a conventional pellet-type catalyst. However, for the tritium removal system, the adsorption process after catalytic oxidation must also employ a honeycomb shape.

Wajima et al. investigated the water vapor adsorption performance of various honeycomb-type adsorbents by changing the temperature, partial pressure of water vapor, and flow rate [12]. In the present study, we focused on the effect of the cell density and zeolite content of the honeycomb-type adsorbents on the water vapor adsorption 
properties and then compared the adsorption performance among test samples from the viewpoint of practical use.

\section{Experimental}

\subsection{Honeycomb-type zeolite}

Figure 1 shows the honeycomb-type and conventional pellet-type zeolites. The honeycomb-type zeolite was prepared by Nagamine Manufacturing Co. Ltd. The specifications of the zeolite samples are summarized in Table 1. The honeycomb adsorbents were prepared by mixing zeolite and a clay binder in specific ratios. The test samples were $20 \mathrm{~mm}$ in diameter and $30 \mathrm{~mm}$ in length. The cells in the honeycomb were square shaped (Fig. 1). In addition, for comparison, 4A molecular sieves with a spherical shape of diameter $2 \mathrm{~mm}$ were purchased from Merck Co. Ltd. as conventional zeolites.
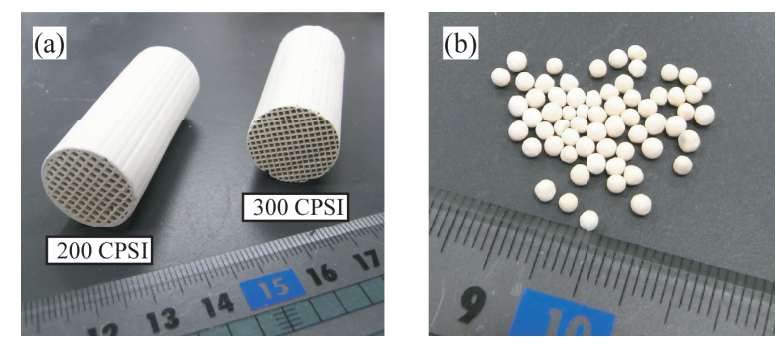

Fig. 1 Photographs of the adsorbents:

(a) honeycomb-type (b) pellet-type. Length of honeycomb-type zeolite is $50 \mathrm{~mm}$.

Table 1 The specifications of adsorbent samples.

\begin{tabular}{c|c|c|c|c}
\hline Shape & $\begin{array}{c}\text { Types of } \\
\text { zeolite }\end{array}$ & $\begin{array}{c}\text { Cell density } \\
{[\mathrm{CPSI}]^{*}}\end{array}$ & $\begin{array}{c}\text { Content of } \\
\text { zeolite }\end{array}$ & Notation \\
\hline \hline Pellet & MS-4A & - & - & P-4A \\
\hline Honeycomb & MS-4A & 300 & $50 \%$ & H-4A-300-50 \\
\hline Honeycomb & MS-4A & 200 & $50 \%$ & H-4A-200-50 \\
\hline Honeycomb & MS-5A & 200 & $50 \%$ & H-5A-200-50 \\
\hline Honeycomb & MS-5A & 200 & $80 \%$ & H-5A-200-80 \\
\hline
\end{tabular}

*CPSI: Cells per Square Inch

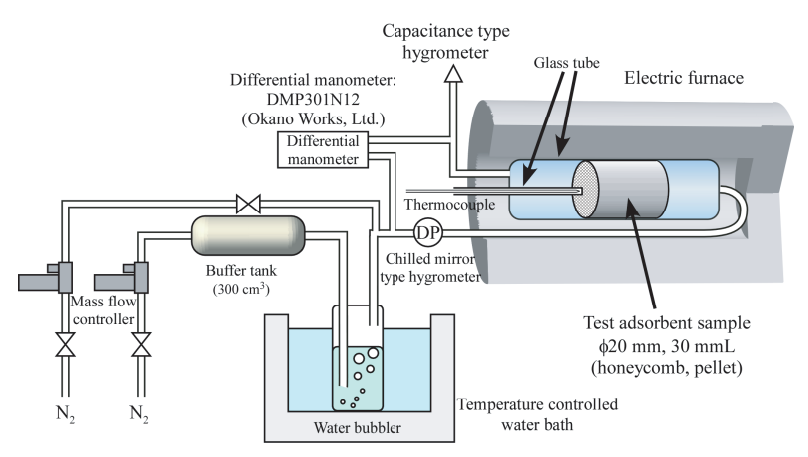

Fig. 2 Schematic diagram of the experimental apparatus.

\subsection{Experimental apparatus and method}

The adsorption properties were evaluated based on the breakthrough method using a flow-type fixed-bed apparatus under various temperatures. Figure 2 shows a schematic diagram of the experimental apparatus. Wet $\mathrm{N}_{2}$ gas was introduced into the test sample at a constant flow rate that was maintained at $307 \mathrm{~cm}^{3} / \mathrm{min}$ by a mass flow controller (Kofloc, Model 3660). The water vapor content in the inlet and outlet of the test sample was measured by a chilled mirror-type hygrometer (GE Sensing, $1111 \mathrm{H}$ ) and a capacitance-type hygrometer (GE Sensing, $\mathrm{M}$ series probe), respectively. The water vapor content in the $\mathrm{N}_{2}$ gas was controlled by a water bubbler immersed in a temperature-controlled water bath. When water vapor with a dew point of $<2{ }^{\circ} \mathrm{C}$ was introduced, the test wet gas was diluted with dry $\mathrm{N}_{2}$ after leaving the water bubbler. The test sample was then heated to about $350^{\circ} \mathrm{C}$ for $3 \mathrm{~h}$ to desorb the residual water under dry $\mathrm{N}_{2}$ gas flow before each experiment. The temperature of the test sample was controlled by an electric heating furnace and was measured by a thermocouple downstream. The adsorption capacity of the adsorbents was estimated to integrate the area between the normalized concentration $c / c_{0}=1$, which is the ratio of the outlet and the inlet water vapor concentration and the breakthrough curve over the entire period.

The pressure drop in the test sample was evaluated by measuring the differential pressure. The test sample used in the pressure drop experiments was $20 \mathrm{~mm}$ in diameter and $50 \mathrm{~mm}$ in length. A differential pressure between the inlet and outlet of the test sample was measured using a differential manometer (Okano Works, Ltd., DMP301N12) at a constant flow rate.

\section{Results and Discussion \\ 3.1 Pressure drop}

Figure 3 shows the pressure drop associated with various adsorbents as a function of linear velocity. The pressure drop observed when the pellet-type sample was used was several times higher than that observed when the honeycomb-type sample was used. These findings indicate that the honeycomb-type sample was suitable for a large processing volume and throughput. It should be noted that no difference in pressure drop was observed among honeycomb samples with different cell densities.

\subsection{Water vapor adsorption properties}

We evaluated the water vapor adsorption properties using breakthrough curves. Figure 4 shows the dependence of water vapor partial pressure on water adsorption capacity. The water vapor partial pressure ranged from 237 to $2074 \mathrm{~Pa}$ at $305 \mathrm{~K}$. The capacity of the test samples to adsorb water vapor increased as the water vapor partial pressure increased. Among these test adsorbents, the pellet-type adsorbent (P-4A) had the largest adsorption capacity for water vapor. The adsorption capacities of the 


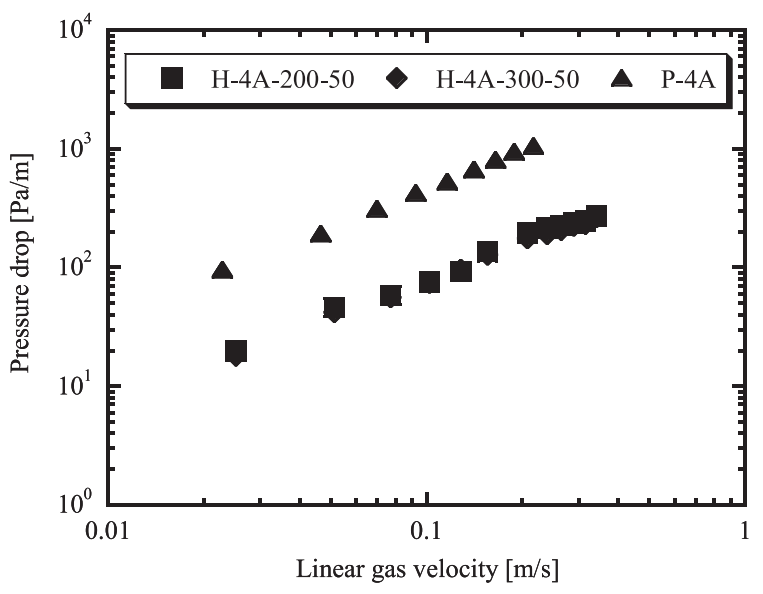

Fig. 3 Effect of the adsorbent shape on pressure drop as a function of linear velocity. The adsorbents were 4A-type zeolite.

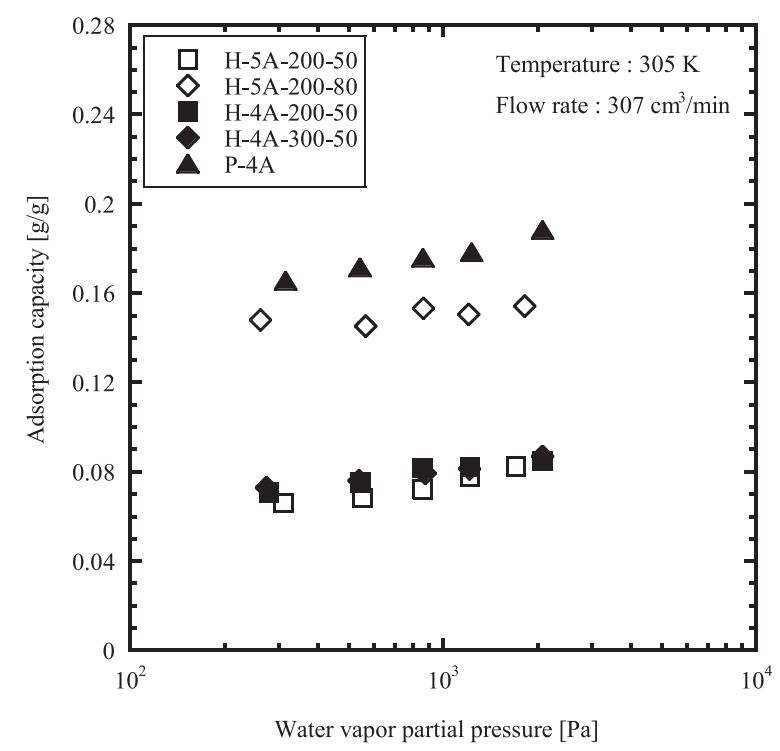

Fig. 4 Dependence of water adsorption capacity on water vapor partial pressure at $305 \mathrm{~K}$.

honeycomb-type adsorbents were lower than that of the pellet type and were proportional to the zeolite content of the honeycomb adsorbents. It should be noted that the cell density did not influence the adsorption capacity.

Figure 5 shows the breakthrough curves for various adsorbents. Although the cell density of the 4A honeycomb-type adsorbent influenced the starting time of the breakthrough curve, there was not much difference between the breakthrough curve starting times for samples $\mathrm{H}$ 4A-200-50, H-4A-300-50, and H-5A-200-50. On the other hand, samples H-5A-200-80 and P-4A had longer breakthrough times, which was probably because they contained a large amount of zeolite. Moreover, the breakthrough curve of the pellet-type adsorbent (P-4A) had a gentler slope than that of the honeycomb adsorbents. These findings indicate that the adsorption rate of the honeycomb-

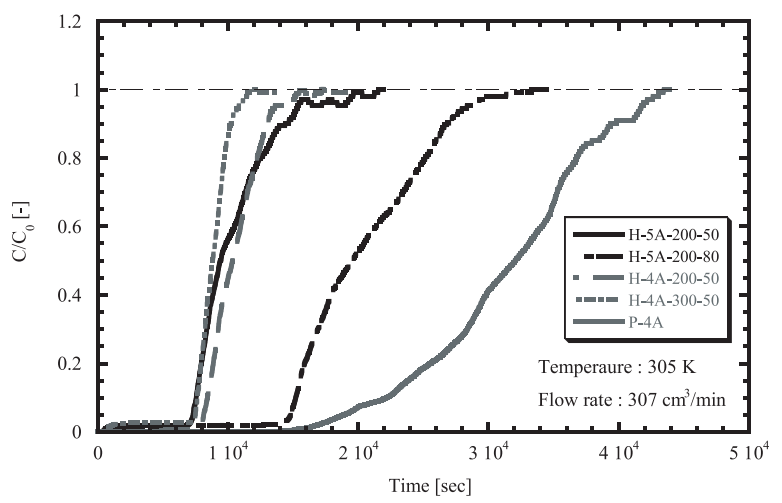

Fig. 5 Breakthrough curves of various adsorbents at $305 \mathrm{~K}$ and a water vapor partial pressure of $1.2 \mathrm{kPa}$.

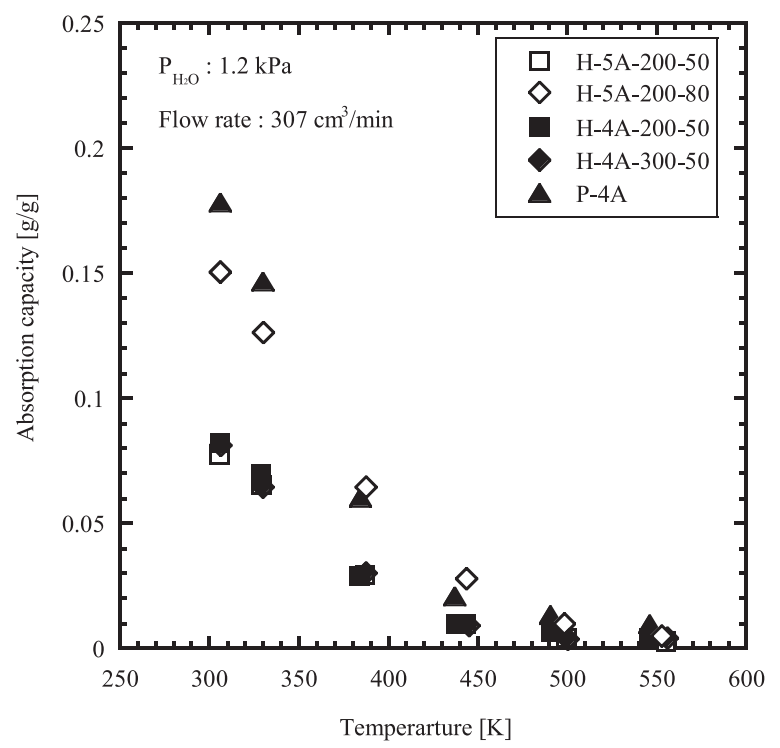

Fig. 6 Dependence of water adsorption capacity on temperature at a water vapor partial pressure of $<1.2 \mathrm{kPa}$.

type zeolite is higher than that of the pellet-type.

During practical use, the adsorbents are periodically regenerated so that they can be reused. During this regeneration, the tritiated water is removed as a condensate. The temperature of the regeneration process is important when determining the optimum conditions. Figure 6 shows the dependence of water adsorption capacity on temperature under $1.2 \mathrm{kPa}$ water vapor partial pressure. As the temperature increased from 305 to $550 \mathrm{~K}$, the adsorption capacity decreased. In particular, the adsorption capacity at temperatures above $450 \mathrm{~K}$ was $<10 \%$ of the capacity at $305 \mathrm{~K}$. In other words, the regeneration process for the honeycombtype zeolite would require a temperature of at least $450 \mathrm{~K}$.

\subsection{Comparison between the adsorption properties of the honeycomb-type zeolite samples}

As mentioned in the previous section, the adsorption capacity of the honeycomb-type adsorbent was pro- 
Table 2 Comparison of the specifications of adsorbent and the adsorption properties.

\begin{tabular}{c||c|c|c|c}
\hline Adsorbent & $\begin{array}{c}\text { H-4A-200- } \\
50\end{array}$ & $\begin{array}{c}\text { H-4A-300- } \\
50\end{array}$ & $\begin{array}{c}\text { H-5A-200- } \\
50\end{array}$ & P-4A \\
\hline \multicolumn{1}{c||}{ Volume $\left[\mathrm{cm}^{3}\right]$} & 5.62 & 4.72 & 5.87 & 7.88 \\
\hline Dry weight [g] & 0.46 & 0.38 & 0.46 & 1.41 \\
\hline $\begin{array}{c}\text { Adsorption weight [g] } \\
305 \mathrm{~K}, 1.2 \mathrm{kPa}-\mathrm{H}_{2} \mathrm{O}\end{array}$ & 0.082 & 0.081 & 0.078 & 0.18 \\
\hline $\begin{array}{c}\text { Adsorption capacity } \\
{[\mathrm{g} / \mathrm{g}]}\end{array}$ & 0.049 & 0.041 & 0.048 & 0.15 \\
\hline $\begin{array}{c}\text { Apparent adsorption } \\
\text { weight }\left[\mathrm{g} / \mathrm{cm}^{3}\right]\end{array}$ & \multicolumn{4}{|c|}{9.4} \\
\hline
\end{tabular}

portional to its zeolite content. These findings indicate that increasing the zeolite content is an effective method of improving the adsorption capacity of the honeycombtype adsorbent. However, the honeycomb-type adsorbent that contained $80 \%$ zeolite was inferior to the adsorbent that contained $50 \%$ zeolite with respect to the precision of molding due to the slight distortion of its cross-sectional shape. The primary cause of this distortion may be compatibility with the binder material and that between the binder and zeolite content. Optimization of the binder material and the molding conditions should be investigated in a future study to enable the solidification of the honeycomb-type zeolite.

The adsorption properties of the honeycomb-type adsorbent samples (except for the sample containing $80 \%$ zeolite) are summarized in Table 2 . The adsorption capacities of the honeycomb-type adsorbents that contained $50 \%$ zeolite were almost the same, despite the zeolite type and cell densities. However, H-4A-200-50 had a larger adsorption capacity per unit volume than $\mathrm{H}-4 \mathrm{~A}-300-50$ because the honeycomb-type zeolite with 200 cells per square inch (CPSI) has thicker walls and higher dry weight than that with 300 CPSI. Therefore, based on their precision of molding and apparent adsorption capacities, H-4A-200-50 and $\mathrm{H}-5 \mathrm{~A}-200-50$ are considered to be suitable for use in a tritium removal system of a fusion power plant.

\section{Conclusion}

For the development of an advanced tritium removal system, the pressure drop and water vapor adsorption properties of various types of honeycomb adsorbents were evaluated at a constant flow rate of $307 \mathrm{~cm}^{3} / \mathrm{min}$. The following results were obtained:

(1) The pressure drop in the honeycomb absorbent was much lower than that in the packed bed-type pellet adsorbent. In addition, the cell density had almost no effect on the pressure drop.

(2) The water vapor adsorption capacity of the test adsorbents increased as the water vapor partial pressure and the zeolite content of the honeycomb adsorbents increased.

(3) The honeycomb-type zeolite with a cell density of 200 CPSI had a larger adsorption capacity per unit volume than that with $300 \mathrm{CPSI}$.

(4) The honeycomb-type zeolite had a higher adsorption rate than the pellet-type zeolite.

(5) The amount of water vapor adsorbed decreased as the temperature increased.

From the viewpoint of practical use, the honeycombtype adsorbent that contained 50\% zeolite with 200 CPSI had superior adsorption properties and a lower pressure drop among the series of adsorbents. The regeneration of honeycomb-type zeolite required a temperature of at least $450 \mathrm{~K}$.

\section{Acknowledgments}

The authors thank Mr. T. Nagamine of Nagamine Manufacturing Co. Ltd. for preparing the honeycomb-type adsorbent. This study was supported by the NIFS budget (NIFS08, 09UCSS004, NIFS10KOBA023) and partially supported by a Grant-in-Aid for Scientific Research (B) (No. 19360416) from the Ministry of Education, Culture, Sports, Science and Technology of Japan.

[1] Radiological control programs for special tritium compounds, DOE-HDBK-1184-2004, U.S. Department of Energy, Washington, (2006), p.2.

[2] Safe handling of tritium: Review of data and experience, Technical Reports Series No. 324, IAEA, Vienna, (1991) p.34.

[3] S. Tanaka and Y. Yamamoto, J. Nucl. Sci. Technol. 13, 251 (1976).

[4] S. Tanaka et al., Fusion Technol. 8, 2196 (1985).

[5] J.E. Nasise, Proc. Tritium Technology in Fission, Fusion and Isotopic Applications (Dayton, Ohio, Apr. 29-May. 1, 1980) p.347.

[6] P. Gildea, Fusion Technol. 8, 2505 (1985).

[7] P. Schirra et al., Fusion Eng. Des. 18, 19 (1991).

[8] M. Yamada et al., Fusion Technol. 28, 1376 (1995).

[9] T. Uda et al., Fusion Sci. Technol. 48, 480 (2005).

[10] T. Uda et al., Fusion Eng. Des. 83, 1715 (2008).

[11] M. Tanaka et al., Fusion Eng. Des. 84, 1818 (2009).

[12] T. Wajima et al., Fusion Eng. Des. 85, 1064 (2010). 